\title{
Using All Sky Cameras to determine cloud statistics for the Thirty Meter Telescope candidate sites
}

Warren Skidmore, Matthias Schöck, Eugene Magnier, David Walker, Dan Feldman, et al.

Warren Skidmore, Matthias Schöck, Eugene Magnier, David Walker, Dan Feldman, Reed Riddle, Sebastian Els, Tony Travouillon, Edison Bustos, Juan Seguel, Joselino Vasquez, Robert Blum, Paul Gillett, Brooke Gregory, "Using All Sky Cameras to determine cloud statistics for the Thirty Meter Telescope candidate sites," Proc. SPIE 7012, Ground-based and Airborne Telescopes II, 701224 (10 July 2008); doi: 10.1117/12.788141

SPIE Event: SPIE Astronomical Telescopes + Instrumentation, 2008, Marseille, France 


\title{
Using All Sky Cameras to determine cloud statistics for the Thirty Meter Telescope candidate sites
}

\author{
Warren Skidmore ${ }^{a}$, Matthias Schöck ${ }^{a}$, Eugene Magnier ${ }^{b}$, David Walker ${ }^{c}$, Dan Feldman ${ }^{d}$, \\ Reed Riddle $^{a}$, Sebastian Els ${ }^{c}$, Tony Travouillon ${ }^{a}$, Edison Bustos ${ }^{c}$, Juan Seguel ${ }^{c}$, Joselino \\ Vasquez $^{c}$, Robert Blum ${ }^{e}$, Paul Gillett ${ }^{a}$, Brooke Gregory ${ }^{c}$ \\ ${ }^{a}$ TMT Observatory Corporation, 2632 East Washington Boulevard, Pasadena, CA 91107, \\ USA; \\ ${ }^{b}$ Institute for Astronomy, 2680 Woodlawn Drive, Honolulu, HI 96822, USA; \\ ${ }^{c}$ AURA/CTIO, Casilla 603, La Serena, Chile; \\ ${ }^{d}$ California Institute of Technology, 1200 East California Boulevard, Pasadena, CA 91125; \\ ${ }^{e} \mathrm{NOAO}, 950$ North Cherry Avenue, Tucson, AZ 85719, USA
}

\begin{abstract}
All Sky Cameras were deployed at all Thirty Meter Telescope (TMT) candidate sites. The images gathered by these cameras were used to assess the cloud statistics for each site. We describe two methods that were developed to do this, a manual method based on inspection of blue and red movies, and an automated method based on photometric analysis of the images.
\end{abstract}

Keywords: Thirty Meter Telescope, site, cloud statistics, clear sky, all sky camera

\section{INTRODUCTION}

Identification of the candidate sites for TMT that were chosen for onsite testing was based on a satellite study of the cloud and precipitable water vapour characteristics and on the expected atmospheric optical turbulence properties, meteorological and logistical considerations of a large number of sites. ${ }^{3}$

Satellite determinations of the cloud coverage are somewhat uncertain due to the assumptions made during the analysis procedure. However, satellite observations covering more a decade are available and some studies have been shown to agree extremely well against naked eye observations. In order to assess the reliability of the satellite observations of clouds above the TMT candidate sites it was necessary to make independent ground based assessments of the cloud statistics over the whole sky at each site that are not affected in any way by the local meteorological conditions.

Historically, naked eye observations are made to determine cloud coverage fractions, but at our remote sites manual observations were not viable. So, as part of the TMT site testing robotic systems, robust All Sky CAmeras (ASCA) were installed at each of the five test sites. A manual method of assessing the cloud category of the ASCA images was developed. Here we describe the manual method developed for cloud assessment using the ASCA images and the confirmation of this technique using line-of-sight Multi Aperture Scintillation Sensor ${ }^{1}$ MASS measurements.

Photometry with telescopes is traditionally used to determine atmospheric optical extinction. Optical atmospheric transparency characteristics encompass both the extinction and cloud characteristics but telescope line-of-sight transparency measurements do not satisfactorily quantify the characteristics of the whole sky for all times. Line-of-sight measurements using the TMT site testing MASS/DIMM (Differential Image Motion

Further author information: (Send correspondence to Warren Skidmore)

Warren Skidmore: E-mail: was@tmt.org, Telephone: +1-626-395-6956

Ground-based and Airborne Telescopes II, edited by Larry M. Stepp, Roberto Gilmozzi,

Proc. of SPIE Vol. 7012, 701224, (2008) · 0277-786X/08/\$18 - doi: 10.1117/12.788141

Proc. of SPIE Vol. 7012 701224-1 
Table 1. The UT dates of beginning of operations of the ASCA cameras on the five TMT candidate sites and the image cycle rate, i.e. the time taken to take a set of images, an image through each of the filters. Some sites had different cycle rates when first installed (Mauna Kea 13N started with 30s cycle time, Tolar and Armazones started with 2 minutes). The Tolar site was closed down on 2007-03-09, all other sites are operational at the time of writing. Final column is the number of 1 hour movies analysed for each site.

\begin{tabular}{l|l|l|l} 
Site & Date $($ YMD) & Image cycle rate & No. of movies \\
\hline Tolar & 20051019 & $4 \mathrm{~min}$ & 2337 \\
Armazones & 20051020 & $4 \mathrm{~min}$ & 4330 \\
Tolonchar & 20051118 & $4 \mathrm{~min}$ & 3083 \\
SPM & 20050601 & $2.5 \mathrm{~min}$ & 3018 \\
Mauna Kea 13N & 20060607 & $4 \mathrm{~min}$ & 2682
\end{tabular}

Monitor $^{2}$ ) systems are problematic because they depend upon the operation of the telescope and instrument. This operation is strongly but not totally linked to weather conditions. If wind, humidity and precipitation measurements from the Automated Weather Station (AWS) are beyond certain limits, the telescope will close down. The telescope also closes down if a star cannot be found, the assumption being that the sky is cloudy, but sometimes errors in the telescope pointing model causes false 'weather pauses'. If the telescope is broken, as has happened both as a result of random hardware failures and due to weather related effects such as lightning strikes, then no line-of-sight measurements are made. The operation of the ASCAs on the other hand is almost totally independent of local weather conditions. We attempted to develop a system that used the ASCA images to do a quantitative transparency and cloud analysis. A system for carrying out photometric analysis of the stellar images in the blue and red ASCA frames was developed and these photometric measurements were analysed. We describe the photometric method that was developed to measure the overall sky transparency but we show that the limitations of this technique precluded its use for the generation of the cloud statistics.

\section{THE ALL SKY CAMERA SYSTEM}

All Sky Cameras (ASCA $)^{4,5}$ were deployed at the five TMT candidate sites. Table 1 lists the dates at which the cameras became operational and the cycle times for a full sequence of images through all filters. At all sites except Mauna Kea 13N, the cameras are mounted on a 3m pillar. The Mauna Kea $13 \mathrm{~N}$ unit is mounted on an arm extending about $1 \mathrm{~m}$ from the Halfmann telescope dome on the top of the $7 \mathrm{~m}$ telescope tower. At San Pedro Mártir (SPM) the ASCA is located about 200m east of the TMT test site, all other ASCAs are located within $20 \mathrm{~m}$ of the MASS/DIMM telescope tower. Images gathered by these cameras cover the whole sky from horizon to horizon.

The red and blue ASCA filters have the following properties:

Red Filter - RG695 (a longpass filter): 630nm cuton - longpass (effectively set by response of CCD)

Blue Filter - BG38 (a bandpass filter): 335nm cuton; 470 midrange; 605 cutoff

\section{THE MANUAL CLOUD ASSESSMENT METHOD}

\subsection{Production of movies}

We have developed a process to create movies that show all quasi-simultaneous blue and red images obtained during each hour. Each image is overlaid by a target pattern, see Figure 1. The name of each movie file identifies the site and UT time of the start of the hour for which the movie was made. Movies were made that included all images obtained up until UT 2008-02-29:23.59, the number of movies for each site is listed in Table 1. 


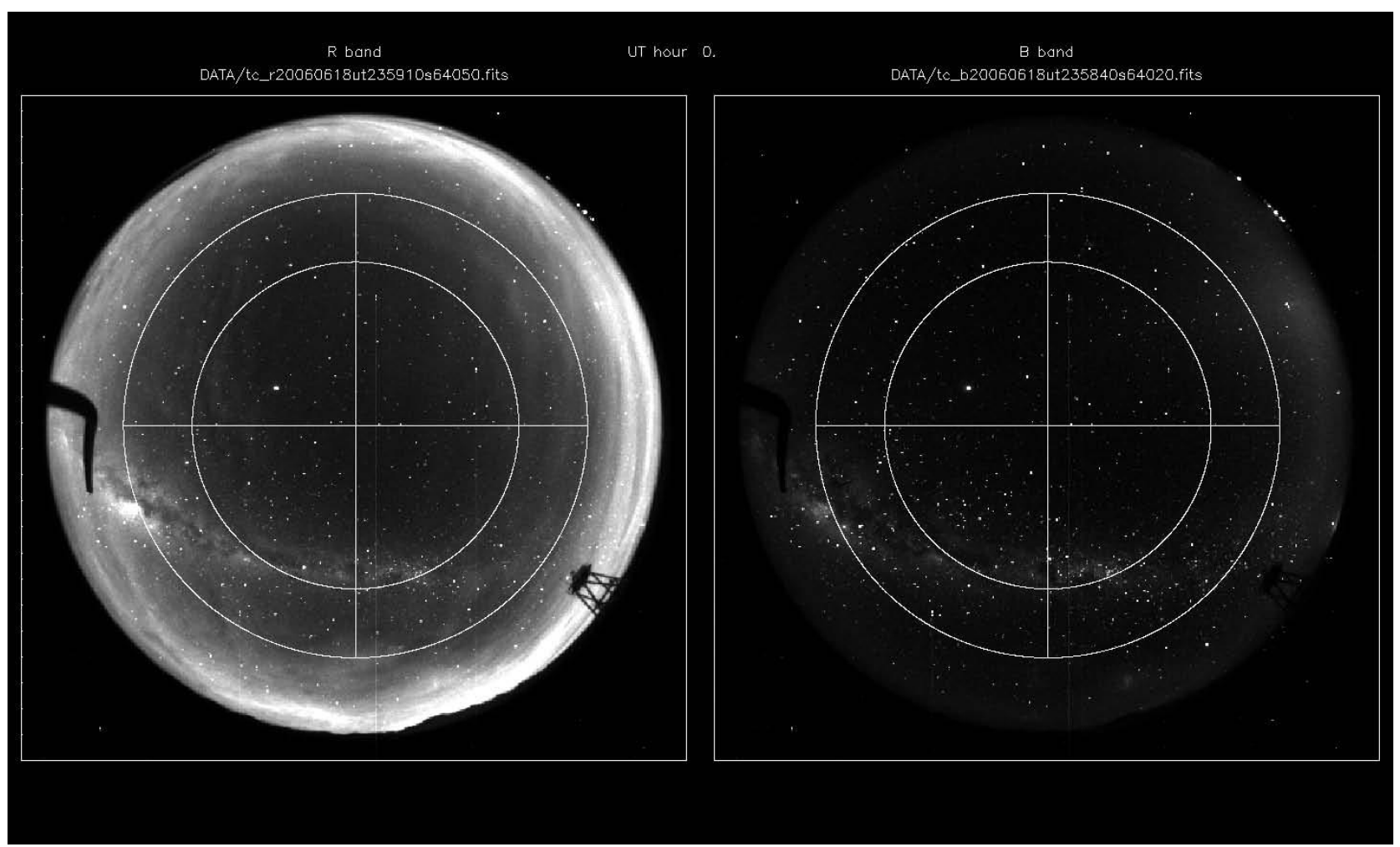

Figure 1. Example of a frame in an ASCA movie. The target is centered on the zenith and has an inner circle and outer annulus of equal area when projected on to the sky, the inner circle covers zenith distance $0^{\circ}$ to $44.7^{\circ}$, the outer annulus goes from $44.7^{\circ}$ to $65^{\circ}$ (the operational limit for TMT). This frame is deliberately chosen to illustrate several issues. Several hot pixels are seen, zodiacal light is seen in the blue image, strong sky glow is seen in the red image, no clouds are visible in these images.

\subsection{Manual categorisation of movies}

A semi-automated script sequentially played each movie and the assessor simply had to press the key corresponding to their assessment of the cloud category for that movie. Two column output files were built up for each site with the UT time for the movie and the corresponding category. Each movie was categorised by an assessor as one of the following:

clear - no cloud inside the target area in either blue or red wavebands

outer - cloud definitely seen in both wavebands in outer target annulus but not in the inner circle

inner - cloud definitely seen in both wavebands in the inner circle

covered - more than $50 \%$ of opaque clouds within the target area, integrated over time and area

moon up - if any of the frames (even a single frame) are short exposure moon up frames

questionable - if unable to make a confident assessment but there is no fundamental problem with the data no data - such as a zero length movie

Cloud outside the target area is ignored. The questionable category is used for example if the the cover over the camera is covered in snow, the camera and images are fine but it is not possible to say whether the sky is cloudy.

All of the ASCA movies for all of the TMT candidate sites were categorised using this method and cloud statistics compiled, see Table 4. 


\subsection{Confidence in the repeatability of the manual categorisation method}

This method is open to problems due to different inerpretations of a movie by different assessors and user errors such as incorrect key strokes (30,000 separate categorisations were made). Assignment of the different categories is somewhat subjective, mis-identification of sky glow as clouds or thin clouds as sky glow can occur, the decision to assign the covered category requires a judgement by the assessor about the degree of cloud and bad pixels and differences in sensitivity between the different cameras may make the detection of thin clouds somewhat site dependent.

By comparing assessments of the same movies by different assessors and comparing those assessments against line-of-sight measurements (see Section 4), the categorisation scheme was improved and modified until it was robust and resulted in uncertainties in the assessments that were well within the acceptable limits needed for site comparison. This scheme had strict categorisation rules that were chosen to minimise the uncertainty in the descisions and relied on appropriate training of each assessor. Repeatability of $1 \%$ was found for all sites except Tolonchar, where the repeatability was $4 \%$. The categorisation scheme is not necessarily the best one for assessing the true usability of a single site but due to the strict categorisation rules and high level of repeatability the scheme allows for a good comparison between sites.

Detection of thin cirrus cloud is an important issue as thin cirrus affects the operation of laser adaptive optics (AO) systems due to back scatter and attenuation of the laser beam. The Keck laser AO system is used in conditions where the extinction is 1 magnitude or better. The ASCA camera at Mauna Kea 13N has the lowest sensitivity of all of our ASCA units. On Mauna Kea there are several laser AO systems (Gemini North, Subaru and Keck) the and we can easily detect the laser beams from these systems in our ASCA images. During periods when the laser AO systems are running we sometimes detect significant levels of clouds that covered the entire sky but did not appear to impact the AO operations (i.e. the AO system continues to operate). Thus we are confident that any clouds that might have an impact on laser AO operations are well within the detection limits of our method.

\subsection{Potential biases of the manual cloud assessment method}

Instrument down times (normally due to equipment failures) are usually uncorrelated with clouds, but there are exceptions. Snow on the site power system solar panels, ${ }^{6,7}$ shutdowns due to the SPM winter break or weather damage (e.g. lightning) preferentially happen during seasons with above-average cloud cover. This means that missed nights might contain more clouds than average conditions which biases toward increasing clear fraction. Snow or condensation on the cameras makes a cloud analysis impossible. This happens more often during bad weather (cloudy) periods than during good weather. This also biases toward increasing clear fraction. Missed data due to bad weather mostly affect Tolonchar, San Pedro Mártir and Mauna Kea 13N. The ASCA CCD at Mauna Kea $13 \mathrm{~N}$ is less sensitive than at other sites, so we are more likely to miss thin clouds, biasing toward increasing clear fraction for Mauna Kea $13 \mathrm{~N}$.

Movies have to be excluded when the moon is up. The fraction of movies missed is larger in the summer (shorter nights) than in the winter, this biases toward decreasing clear fraction at all sites (except maybe Tolonchar, which also has a cloudy period in the summer, the Bolivian winter).

It is not clear whether sky glow causes a bias increasing the clear or cloudy fraction. Sometimes thin clouds are missed because of sky glow. Sometimes sky glow is mistaken for clouds. It is also not clear from watching the movies if sky glow is, on average, stronger at some sites than others.

After watching lots of movies, we believe that the combined effect of the competing biases is small and within the inherent uncertainty of the analysis. We do, however, probably miss a few more clouds at Tolonchar, 
Table 2. The statistical comparison of the sum of the ASCA clear and outer categories versus MASS LOSSAM values less than 0.06 for simultaneous data. Note: The MASS only operates during clear conditions so the clear fractions reported here are gross over-estimates.

\begin{tabular}{l|c|c|c|c} 
& ASCA & MASS $<0.06$ & Difference & Site elevation \\
\hline Tolar & $84.8 \%$ & $84.4 \%$ & $0.4 \%$ & $2290 \mathrm{~m}$ \\
SPM & $80.1 \%$ & $81.6 \%$ & $-1.5 \%$ & $2830 \mathrm{~m}$ \\
Armazones & $88.0 \%$ & $85.1 \%$ & $2.9 \%$ & $3064 \mathrm{~m}$ \\
Mauna Kea 13N & $86.7 \%$ & $81.2 \%$ & $5.5 \%$ & $4050 \mathrm{~m}$ \\
Tolonchar & $80.7 \%$ & $74.5 \%$ & $6.2 \%$ & $4475 \mathrm{~m}$
\end{tabular}

San Pedro Mártir and Mauna Kea 13N than at Armazones and Tolar.

\section{CROSS CHECKS OF THE MANUAL CLOUD ANALYSIS AGAINST THE LOSSAM METHOD USING THE MASS DEVICE}

The Multi-Aperture Scintillation Sensor (MASS) is described in [1]. A MASS measures the flux coming from a bright star with four photo multipliers (one for each of the four MASS subapertures) at a frequency of 1000 Hz. Short-term variations of the flux can therefore be detected with high accuracy. In principle, one could also use the total flux received by the MASS as a measure of extinction. In practice, this is complicated by effects such as the accumulation of dust on the optics. Instead, the LOSSAM (Line Of Sight Sky Absorption Monitor) method is used. This method was developed for the VLT Astronomical Site Monitor at Cerro Paranal. ${ }^{8}$ It is based on the observation that the root-mean-square (rms) fluctuations of the flux from a bright star, averaged over several minutes and divided by the mean flux, are well correlated with the atmospheric extinction coefficient. As one second rms and mean fluxes of the MASS measurements are saved to disk and archived for all our sites, the atmospheric extinction can thus be calculated in post-processing for all times for which MASS data are available. Ten minute sets of the one second RMS fluctuations are averaged. If the telescope changes star during this time the ten minute set is excluded from further analysis. All valid ten minute sets corresponding to a one hour ASCA movie category are averaged to give a mean LOSSAM value for that hour. We compared the LOSSAM values derived from the MASS with simultaneous ASCA categories for a range of LOSSAM values. The LOSSAM value that gave the closest match between simultaneous MASS and ASCA images is 0.06 . i.e. clear $<0.06<$ cloudy.

The overall statistical match for all five candidate sites is very good, see Table 2 . There may be a slight dependence on altitude which could be due to an altitude effect on the LOSSAM measurements. The statistical values are the fraction of clear categories for all of the ASCA or all of the MASS measurements that are simultaneous. Obviously the MASS/ASCA comparison is based on the chosen value for the LOSSAM cut off but the key point is that the two methods of measuring the effect and presence of cloud correlate strongly.

\section{THE PHOTOMETRIC EXTINCTION SOFTWARE}

An image analysis system to make photometric measurements of stars was set up for the ASCAs that was based on the system developed first for the CFHT MegaCam $\left(0.96^{\circ}\right.$ by $0.94^{\circ}$ fov $)$ and then modified for the Pan-STARRS system (with $3^{\circ}$ by $3^{\circ}$ fov).

The system works by identifying potential stellar images in each frame and, based on the expected star pattern for that sidereal time and location, astrometrically matches those images to a catalogue of known stars. The measured output magnitudes and position in RA. and Dec., catalogue magnitudes, etc., for each star and each frame are stored in a database. This data base can be queried to provide relevant measurements based on time and/or stellar identification. 
The astrometric matching fails when insufficient stars are detected, so the scheme is fundamentally limited to operation in relatively clear conditions. However, sometimes for apparently good ASCA images, the system would fail to converge on an astrometric solution or would generate an incorrect astrometric solution. So, a lack of data in the data base corresponding to a particular ASCA image usually but not exclusively indicates significant amounts of cloud.

Based on the output of the above photometric analysis system, an automated method was developed for determining the extinction at the position on the sky of each stellar detection in every single blue and red ASCA image using $\mathrm{M}_{\text {extinction }}=\mathrm{M}_{\text {catalogue }}-\mathrm{M}_{\text {measured }}$. By averaging the extinction values over areas corresponding to the target areas described in Figure 1, this allows a comparison with the manual method.

Each individual stellar photometric measurement has a typical signal to noise ratio of about 10, and across an entire clear sky image there are up to a few thousand detections. Signal to noise of each individual photometric measurement ss fundamentally limited by the spatial sensitivity variations across the CCD. The angular scale projected onto the CCD of $0.19^{\circ}$ per pixel means that large fractions of the stellar flux can fall between pixels and spatial sensitivity variations within individual pixels become significant. For clear sky images, systematic noise sources dominate the noise terms for most of the sources detected, only a few percent of the faintest detected sources have photon noise components that begin to approach the systematic effects. Due to the large number of stars typically detected the statistical uncertainty on the averaged extinction value for a particular image is very small.

However this automated method was badly affected by additional systematic problems that introduce trends into the averaged extinction values and limit the subsequent cloud categorisations to an unacceptable level. Systematic problems include but are not limited to: the accumulation of dust on the perspex camera cover and periodic cleaning, flat field issues, repositioning of the camera after maintenance and mis-identification of the stars during image processing causing discrete jumps around $10 \%$ in the catalogue magnitude assigned to to a particular star. Figure 2 demonstrates these systematic effects in the measured mean hourly extinctions for blue and red images. Groups of 'clear' points (dots) indicate the clear sky extinction, and variations in the 'clear' extinction level clearly span the range in which some periods are classed as having clouds in the manual cloud analysis method. Clearly, the correlation between the manual cloud analysis and automatic extinction measurements is good on the short term, but the large amplitude longer term systematic variations invalidate any categorisation based on the extinction levels.

Table 3 shows the large uncertainties remaining in the derived extinction values for the Tolar and Mauna Kea $13 \mathrm{~N}$ sites. One hour mean extinction values are derived from all blue and red band stellar detections for a particular hour. Separate values are derived corresponding to the inner, outer and whole target area as used in the manual method. The average and standard deviation around the average of all of the one hour means corresponding to the four valid manual cloud categories and the three target areas are reported here. A clear correlation is seen in the measured extinction as we go from clear to cloudy. This analysis only includes those images for which a valid astrometric solution was obtained.

Despite exhaustive attempts to deal with the systematic variations in the measured extinction and also derive a method for coping with the frames for which the photometric analysis failed, the automated method was ultimately unable to yield the required level of accuracy for site comparison based on the measured extinctions or derived cloud characteristics.

\section{CONCLUSIONS}

The manual cloud analysis method is reported to be highly repeatable amongst different assessors and shown to correlate well with MASS LOSSAM measurements. The automated extinction method that was developed 


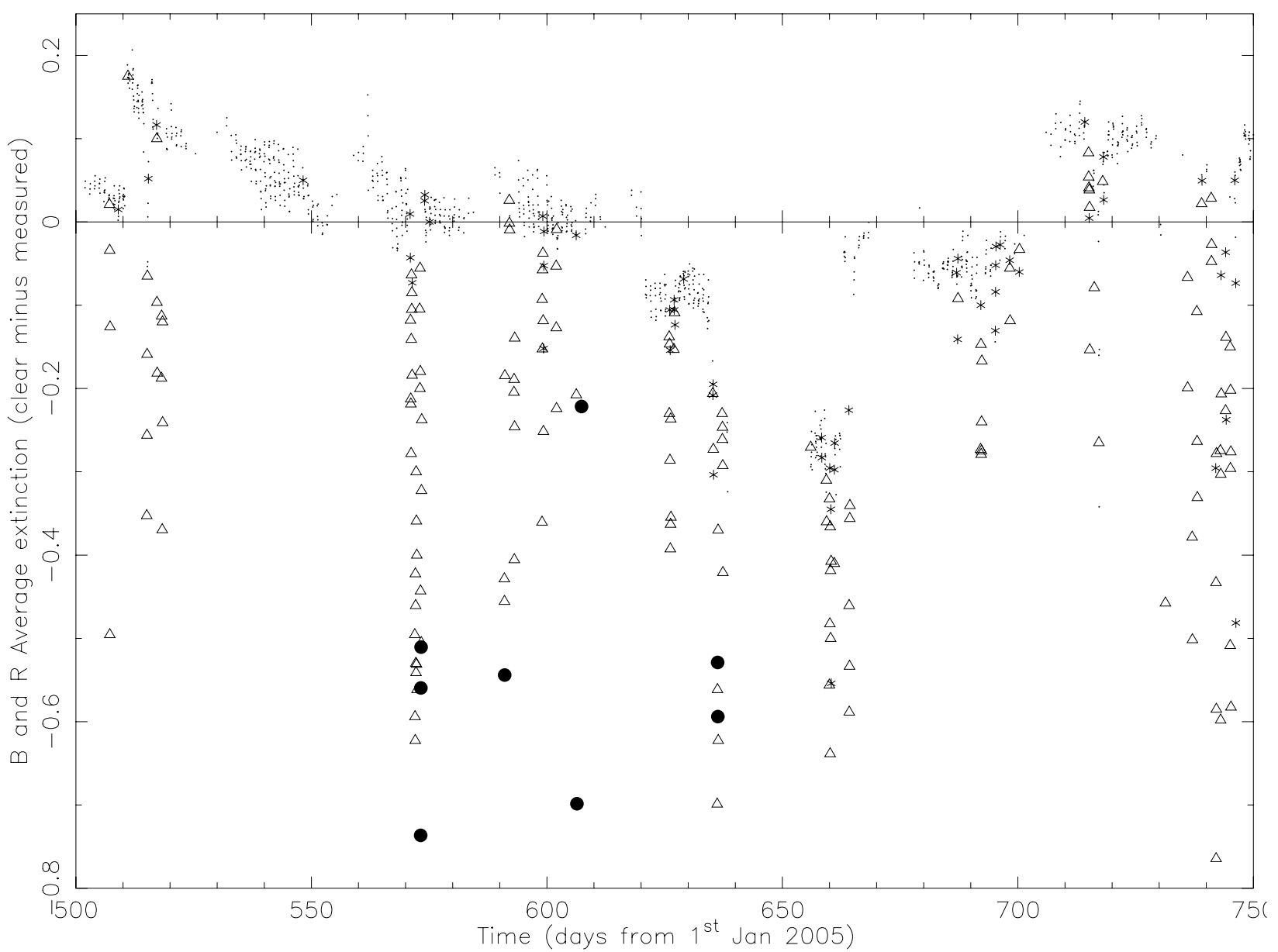

Figure 2. Plot to show the systematic effects on the mean hourly blue and red extinction for the inner target area. Each point represents the mean extinction for all stellar detections in all blue and red images in a particular hour. The corresponding manual cloud category is indicated by the point shape, dots indicate the 'clear' manual category, stars indicate 'outer', triangles are 'inner', filled circles are the 'cloudy' category.

Table 3. The mean extinction in magnitudes $\left(\mathrm{M}_{\text {clear }}-\mathrm{M}_{\text {measured }}\right)$ and standard deviation (1 $\sigma$ error bar) around the mean for the automated photometric extinction method values corresponding to the four manual cloud categories and three target areas. Identical analysis was done for all sites.

\begin{tabular}{c|c|c|c|c|c|c} 
& \multicolumn{3}{|c|}{ Tolar } & \multicolumn{3}{c}{ Mauna Kea 13N } \\
\hline & All & Outer & Inner & All & Outer & Inner \\
\hline Clear & $-0.003 \pm 0.063$ & $-0.025 \pm 0.048$ & $-0.021 \pm 0.087$ & $-0.075 \pm 0.041$ & $-0.078 \pm 0.033$ & $-0.073 \pm 0.052$ \\
Outer & $-0.06 \pm 0.10$ & $-0.076 \pm 0.085$ & $-0.05 \pm 0.13$ & $-0.16 \pm 0.11$ & $-0.147 \pm 0.088$ & $-0.18 \pm 0.14$ \\
Inner & $-0.19 \pm 0.16$ & $-0.17 \pm 0.13$ & $-0.22 \pm 0.21$ & $-0.25 \pm 0.14$ & $-0.22 \pm 0.11$ & $-0.29 \pm 0.17$ \\
Cloudy & $-0.44 \pm 0.12$ & $-0.33 \pm 0.12$ & $-0.62 \pm 0.22$ & $-0.36 \pm 0.15$ & $-0.32 \pm 0.13$ & $-0.42 \pm 0.18$
\end{tabular}


Table 4. The cloud statistics derived from the ASCA movies. These numbers may not be representative of the long term cloud statistics for each site due to the restricted time span for the measurements (see Table 1).

\begin{tabular}{l|l|l|l|l} 
& Clear & Outer & Inner & Covered \\
\hline Tolar & $77.4 \%$ & $6.3 \%$ & $14.5 \%$ & $1.8 \%$ \\
Armazones & $82.5 \%$ & $4.3 \%$ & $9.3 \%$ & $3.9 \%$ \\
Tolonchar & $72.4 \%$ & $5.4 \%$ & $10.4 \%$ & $11.8 \%$ \\
SPM & $72.6 \%$ & $5.3 \%$ & $11.5 \%$ & $10.6 \%$ \\
MK 13N & $70.9 \%$ & $6.5 \%$ & $9.8 \%$ & $12.8 \%$
\end{tabular}

is shown to correlate with the manual cloud analysis method, but systematic effects on the derived extinction values cause uncertainties that are larger than the accuracy required to compare sites.

The cloud statistics derived using the manual assessment method of the ASCA observations for each site are listed in Table 4.

\section{ACKNOWLEDGMENTS}

The authors acknowledge the contributions of the late Hugo Schwarz to the development, construction and deployment of the ASCA units and his assistance during the interpretation of the ASCA images.

The authors gratefully acknowledge the support of the TMT partner institutions. They are the Association of Canadian Universities for Research in Astronomy (ACURA), the California Institute of Technology and the University of California. This work was supported as well by the Gordon and Betty Moore Foundation, the Canada Foundation for Innovation, the Ontario Ministry of Research and Innovation, the National Research Council of Canada, the Natural Sciences and Engineering Research Council of Canada, the British Columbia Knowledge Development Fund, the Association of Universities for Research in Astronomy (AURA) and the U.S. National Science Foundation.

The San Pedro Mártir ASCA system is owned and operated by the LSST project and data is made available to TMT as part of a data sharing agreement.

The LSST design and development activity is supported by the National Science Foundation under Scientic Program Order No. 9 (AST-0551161) through Cooperative Agreement AST-0132798, and at NOAO under AST-0244680. Additional funding comes from private donations, in-kind support at Department of Energy laboratories and other LSSTC Institutional Members.

\section{REFERENCES}

[1] Els S., et. al., Study on the precision of the MASS turbulence profiler employed in the site testing campaign for the Thirty Meter Telescope, 2008, Applied Optics, in press

[2] Wang, L., et. al., High-accuracy differential image motion monitor measurements for the Thirty Meter Telescope site testing program, 2007, Appl. Opt. 46, 6460-6468

[3] Thirty Meter Telescope Construction Proposal, 2007, http://www.tmt.org/news/TMT-Construction\%20Proposal-Public.pdf

[4] Walker D., Schwarz H., Bustos E., Monitoring the night sky with the Cerro Tololo All-Sky camera for the TMT and LSST projects, 2006, SPIE, 6267, P. 87, ed. Stepp L.

[5] Riddle R., et. al., An analysis of light pollution at the Thirty Meter Telescope candidate sites, 2008, SPIE, 7012-74, in press, ed. Stepp L.

[6] Riddle R., Schöck M., Skidmore W., The Thirty Meter Telescope site testing robotic computer system, 2006, SPIE, 6267, P. 56, ed. Stepp L. 
[7] Skidmore W., et. al., The Thirty Meter Telescope site testing system, 2004, SPIE 5489, P. 154, ed. Oschmann J.

[8] Sarazin M., http://www.eso.org/gen-fac/pubs/astclim/lasilla/asm/lossam/ 2000, ESO public pages 